\title{
SVM and Neural Network based Diagnosis of Diabetic Retinopathy
}

\author{
R.Priya \\ Lecturer [Selection Grade] \\ Department of CSE \\ Annamalai University \\ Chidambaram
}

\author{
P. Aruna \\ Associate Professor \\ Department of CSE \\ Annamalai University \\ Chidambaram
}

\begin{abstract}
Diabetic retinopathy (DR) is an eye disease caused by the complication of diabetes and we should detect it early for effective treatment. As diabetes progresses, the vision of a patient may start deteriorate and lead to diabetic retinopathy. As a result, two groups were identified, namely nonproliferative diabetic retinopathy (NPDR) and proliferative diabetic retinopathy (PDR). In this paper, to diagnose diabetic retinopathy, two models like Probabilistic Neural network (PNN) and Support vector machine (SVM) are described and their performances are compared. Experimental results show that PNN has an accuracy of $89.60 \%$ and SVM has an accuracy of $97.608 \%$. This infers that the SVM model outperforms the other model.
\end{abstract}

\section{Keywords}

Diabetic Retinopathy, Probabilistic Neural network, Support vector machine, Sensitivity, Specificity.

\section{INTRODUCTION}

Diabetes is a group of metabolic diseases in which a person has high blood sugar, either because the body does not produce enough insulin, or because cells do not respond to the insulin that is produced.[1] Diabetic retinopathy is one of the common complications of diabetes. It is a severe and widely spread eye disease. It damages the small blood vessels in the retina resulting in loss of vision. The risk of the disease increases with age and therefore, middle aged and older diabetics are prone to Diabetic Retinopathy. Nonproliferative diabetic retinopathy is an early stage of diabetic retinopathy. In this stage, tiny blood vessels within the retina leak blood or fluid. The leaking fluid causes the retina to swell or to form deposits called exudates. Proliferative diabetic retinopathy, PDR is an attempt by the eye to grow or re-supply the retina with new blood vessels (neovascularization), due to widespread closure of the retinal blood supply.[2]

\section{RELATED WORK}

During the recent years, there have been many studies on automatic diagnosis of diabetic retinopathy using several features and techniques. D.Vallabha et al. [3] proposed a method for automated detection and classification of vascular abnormalities in Diabetic Retinopathy using scale and orientation selective Gabor filter banks. R.Sivakumar et al. [4] presented a method to classify diabetic retinopathy subjects from changes in visual evoked potential spectral components. According to Thomas Walter et al. [5] exudates are found using their high grey level variation, and their contours are determined by means of morphological reconstruction techniques. HT Nguyenl et al. [6], proposed a multilayer feed forward network for the classification of DR. A. M. Mendonça, et al. [7] proposed a method in which microaneurysms are validated using two new criteria, based on local intensity, contrast and shape relations. Huiqi $\mathrm{Li}$ and Opas Chutatape [8] describes the development of an automated fundus image processing system to facilitate diagnosis of the ophthalmologists. María García et al. [9] used a multilayer perceptron (MLP) classifier to obtain a final segmentation of HEs in the image. Kenneth W. Tobin et al. [10] developed a content-based image retrieval method to verify the hypothesis that retinal pathology can be identified and quantified from visually similar retinal images in an image archive. Chaudhury.S et. al [11] address the problem of detecting blood vessels in retinal images. They have used the concept of matched filter for detection of signals to detect piecewise linear segments of blood vessels in retinal images and constructed 12 different templates to search for vessel segments along all possible directions. Jie Tian et al. [12] used a probabilistic neural network (PNN) as a classifier to the automatic classification of underwater objects. In [13], Katia Estabridis and Rui J. P. de Figueiredo detects the fovea, blood vessel network, optic disk, as well as bright and dark lesions associated with DR. V. Vijaya Kumari et al. in [14] detected many of the features such as blood vessel, exudates and optic disk accurately using morphological operations. In [15] Alireza Osareh et al. classified the segmented regions into two disjoint classes, exudates and non-exudates, comparing the performance of various classifiers. Jian $\mathrm{Wu}$ et.al [16] proposed a cerebral aneurysm recognition method using Bayesian classification. In [17], Yosawin Kangwanariyakul et al. proposed to use Back-propagation neural network (BPNN), the Bayesian neural network(BNN), the probabilistic neural network (PNN) and the support vector machine (SVM)were applied to develop classification models for identifying IHD patients. In [18], PV Nageswara rao et al. proposed a new approach for protein classification based on a Probabilistic Neural Network and feature selection. In [19], Inan Guler and Elif Derya U beyli proposed the probabilistic neural network (PNN) and multilayer perceptron neural network for the classification of the EEG signals. Lili $\mathrm{Xu}$ and Shuqian Luo in [20] used a support vector machine (SVM) with Gaussian radial basis function as a classifier to identify hard exudates from digital retinal images. In [21], Priya.R and Aruna.P used SVM for the detection of diabetic retinopathy stages using color fundus images.

\section{PROPOSED SYSTEM}

In this paper, an automated approach for classification of the disease diabetic retinopathy using fundus images is presented. A fundus camera or retinal camera is a specialized low power microscope with an attached camera designed to photograph the interior surface of the eye, including the retina, optic disc, macula, and posterior pole. [22] The images were captured using a Canon TopCon TRC-50 EX with 
Nicon retinal camera at a field-of-view (FOV) of $50^{\circ}$. The acquired image resolution is $1280 \times 1024$ in 24bit JPEG format. The evaluation of the proposed automated diagnosis system of diabetic retinopathy have been performed by using a set of 250 images which is a combination of normal, NPDR and PDR affected images. The original image which is of size $1280 \times 1024$ is converted to gray scale image. After that, adaptive histogram equalization is applied to improve the contrast of the image. Then, DWT is applied and the size of the image is reduced into half as $640 \times 512$. Then Matched filter response (MFR) is applied to reduce the noise in the image. Finally, Fuzzy c-means clustering is applied to segment the blood vessels in the image. After pre-processing of images is completed, features such as Radius, Diameter, Area, Arc length, Centre Angle and Half area are calculated for each image. Then Modeling Techniques like PNN, Bayes Theory and SVM are used and their performances are compared. Finally, the images are classified into three groups namely, normal image, Non-Proliferative Diabetic Retinopathy (NPDR), and Proliferative Diabetic Retinopathy (PDR). The remainder of this paper is organized as follows. Section 4 describes the preprocessing of images. Section 5 explains the feature extraction. Section 6 describes the classification of DR disease using Support Vector Machine. Section 7 explains Probabilistic Neural Network. Section 8 describes the experimental results. Section 9 gives the conclusion. Figure 1. gives the block diagram of the proposed system for diagnosis of Diabetic Retinopathy.

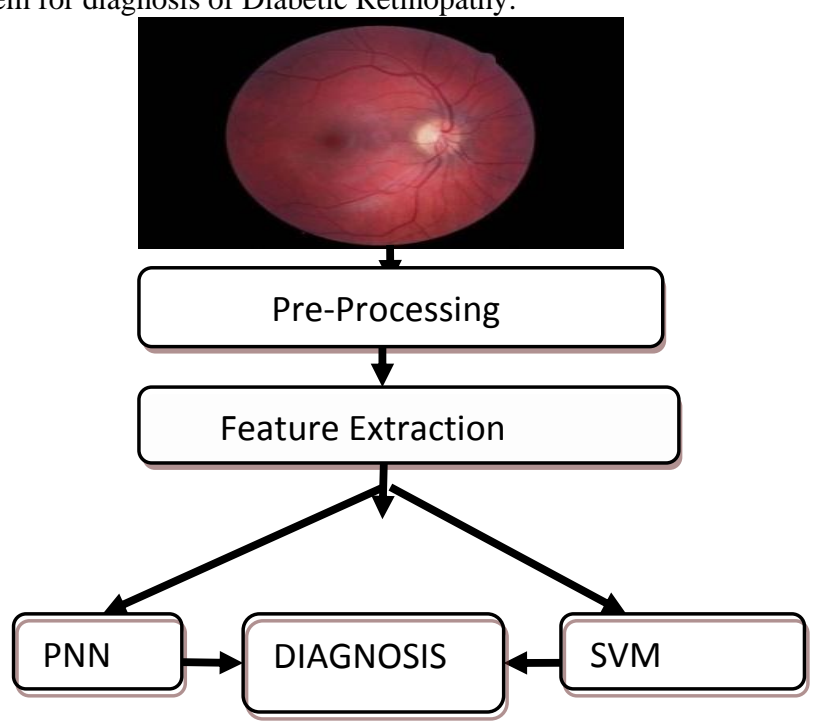

Fig. 1: Block diagram for the comparison between two classifiers for Diagnosis of DR

\section{PREPROCESSING OF IMAGES}

In detecting abnormalities associated with fundus image, the images have to be pre-processed in order to correct the problems of uneven illumination problem. The techniques for preprocessing include Gray scale Conversion, Adaptive Histogram Equalization, Discrete Wavelet Transform, Gaussian Matched Filter Response and Fuzzy C-means Clustering for segmentation of blood vessels. The acquired image resolution is $1280 \times 1024$ in 24bit JPEG format. The color image of an eye is taken as input image and is converted to a grayscale image. Adaptive histogram equalizsation which is used to improve contrast in images, is applied to the gray scale converted eye image. Consider a running sub image $\mathrm{W}$ of $\mathrm{N} X \mathrm{~N}$ pixels centered on a pixel $\mathrm{P}(\mathrm{i}, \mathrm{j})$, the image is filtered to produce another sub image $\mathrm{P}$ of $(\mathrm{N} \mathrm{X} \mathrm{N})$ pixels according to the equation below:

$$
P_{n}=255\left(\frac{\left[\phi_{w}(p)-\phi_{w}(\operatorname{Min})\right]}{\left[\phi_{w}(\operatorname{Max})-\phi_{w}(\text { Min })\right]}\right)
$$

Where

$\phi_{w}(P)=\left[1+\exp \left(\frac{\mu_{w}-p}{\sigma_{w}}\right)\right]^{-1}$

and Max and Min are the maximum and minimum intensity values in the, whole eye image while $\mu_{\mathrm{w}}$ indicate the local window mean and $\sigma_{\mathrm{w}}$ indicate standard deviation which are defined as:

$$
\begin{aligned}
\mu_{w} & =\frac{1}{N^{2}} \sum_{(i, j) \varepsilon(k, l)} P(i, j) \\
\sigma_{w} & =\sqrt{\frac{1}{N^{2}} \sum_{(i, j) \varepsilon(k, l)}\left(P(i, j)-\mu_{w}\right)^{2}}
\end{aligned}
$$

As a result of this adaptive histogram equalisation, the dark area in the input eye image that was badly illuminated has become brighter in the output eye image while the side that was highly illuminated remains or reduces so that the whole illumination of the eye image is same. The transform of a signal is just another form of representing the signal. It does not change the information content present in the signal. The Discrete Wavelet Transform (DWT), which is based on subband coding, is found to yield a fast computation of Wavelet Transform. It is easy to implement and reduces the computation time and resources required. Wavelet transform decomposes a signal into a set of basis functions. These basis functions are called wavelets. Wavelets are obtained from a single prototype wavelet $\psi(\mathrm{t})$ called mother wavelet by dilations and shifting:

$$
\psi_{a, b}(t)=\frac{1}{\sqrt{a}} \psi\left(\frac{t-b}{a}\right)
$$

where ' $a$ ' is the scaling parameter and ' $b$ ' is the shifting parameter. The mother wavelet used to generate all the basis functions is designed based on some desired characteristics associated with that function. $[\mathrm{cA}, \mathrm{cH}, \mathrm{cV}, \mathrm{cD}]=$ dwt2(X,'wname') computes the approximation coefficients matrix $\mathrm{cA}$ and details coefficients matrices $\mathrm{cH}, \mathrm{cV}$, and $\mathrm{cD}$ (horizontal, vertical, and diagonal, respectively), obtained by wavelet decomposition of the input matrix $\mathrm{X}$ where $\mathrm{X}$ is the given input eye image after applying adaptive histogram equalization. The 'wname' string contains the wavelet name. In this paper, Haar wavelet is used. As a result of applying this DWT to the eye images, the size of the images are reduced to half. So size of the eye images are now $640 \times 512$. The matched filter is the optimal linear filter for maximizing the signal to noise ratio (SNR) in the presence of additive stochastic noise. The optimal filter is given by

$$
h_{\text {opt }}(d)=-\exp \left(-d^{2} / 2 \sigma^{2}\right)
$$


The negative sign indicates that the vessels are darker than the background. Also, instead of ' $n$ ' different types of objects having to be identified, the problem reduces to deciding whether or not a particular pixel belongs to a blood vessel. If the magnitude of the filtered output at a given pixel location exceeds a certain threshold, the pixel is labeled as a part of a vessel. When the concept of matched filter is extended to two dimensional images, it must be appreciated that a vessel may be oriented at any angle $\theta(0 \leq \theta \leq \pi / 2)$. The matched filter $\mathrm{s}(\mathrm{t})$ will have its peak response only when it is aligned at an angle $\theta \pm \pi / 2$. Thus, the filter needs to be rotated for all possible angles, the corresponding responses are to be compared, and for each pixel only the maximum response is to be retained. Consider the response of this filter for a pixel belonging to the background retina. Assuming the background to have constant intensity with zero mean additive Gaussian white noise, the expected value of the filter output should ideally be zero. Instead of matching a single intensity profile of the cross section of a vessel, a significant improvement can be achieved by matching a number of cross sections (of identical profile) along its length simultaneously. Such a kernel may be mathematically expressed as

$\mathrm{K}(\mathrm{x}, \mathrm{y})=-\exp \left(-\mathrm{x}^{2} / 2 \sigma^{2}\right)$

for $|y| \leq L / 2$

Where $L$ is the length of the segment for which the vessel is assumed to have a fixed orientation. Here the direction of the vessel is assumed to be aligned along the y-axis. For the vessels at different orientations, the kernel has to be rotated accordingly. As a result of applying this MFR to retinal images, response due to the noise is suppressed significantly, where no blood vessel is present. Fuzzy c-means (FCM) is a method of clustering which allows one piece of data to belong to two or more clusters. Here it is used to segment the input eye image and detect the blood vessels. Information about blood vessels can be used in grading disease severity or as part of the process of automated diagnosis of diseases with ocular manifestations. It is based on minimization of the following objective function:

$$
J_{m}=\sum_{i-1}^{N} \sum_{j-1}^{C} u_{i j}^{m}\left\|x_{i}-c_{j}\right\|^{2}, 1 \leq \mathrm{m}<\alpha
$$

where $m$ is any real number greater than $1, u_{i j}$ is the degree of membership of $x_{i}$ in the cluster $j, x_{i}$ is the ith of d-dimensional measured data, $c_{j}$ is the d-dimension center of the cluster, and $\|*\|$ is any norm expressing the similarity between any measured data and the center. The algorithm is composed of the following steps:

1. Initialize $\mathrm{U}=\left[\mathrm{u}_{\mathrm{ij}}\right]$ matrix, $\mathrm{U}(0)$

2. At $\mathrm{k}$-step: calculate the centers vectors $\mathrm{C}(\mathrm{k})=\left[\mathrm{c}_{\mathrm{j}}\right]$ with $\mathrm{U}(\mathrm{k})$

$c_{j}=\frac{\sum_{i-1}^{N} u_{i j}^{m} \cdot x_{i}}{\sum_{i-1}^{N} u_{i j}^{m}}$

3. Update $\mathrm{U}(\mathrm{k}), \mathrm{U}(\mathrm{k}+1)$

$$
u_{i j}=\frac{1}{\sum_{k-1}^{c}\left(\frac{\left\|x_{i}-c_{j}\right\|}{\left\|x_{i}-c_{k}\right\|}\right)^{\frac{2}{m-1}}}
$$

4. If $\|\mathrm{U}(\mathrm{k}+1)-\mathrm{U}(\mathrm{k})\|<\varepsilon$ then STOP; otherwise return to step 2.

The Original DR affected eye Images and the resulting images after preprocessing are shown in Figure 2. below.

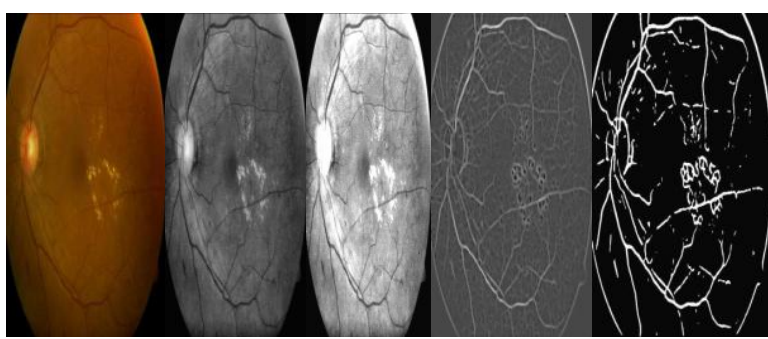

(a)

(b)

(c)

(d)

(e)

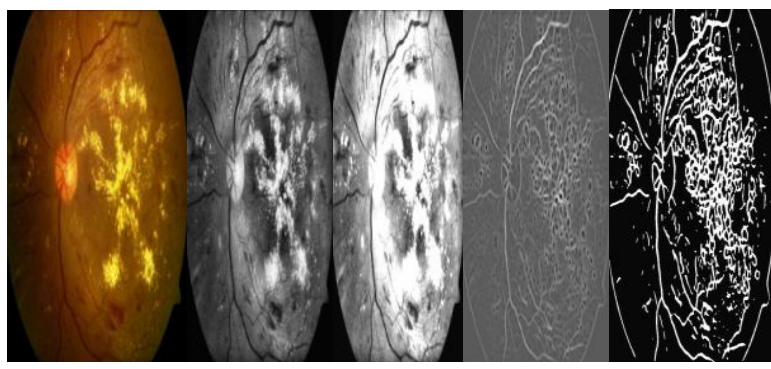

(f) $\quad($ g) $\quad$ (h) $\quad$ (i)

(j)

Fig. 2: (a) NPDR affected Eye Image (f) PDR affected Eye Image (b),(g) Histogram Equalization (c),(h) Discrete Wavelet Transform (d),(i) Matched Filter Response (e),(j) Fuzzy C-means segmentation

\section{FEATURE EXTRACTION}

After performing the above mentioned preprocessing steps, the new eye image is obtained. Since the retinal image is of shape circle, features related to circle are taken. The features that are extracted are: Radius, Diameter, Area, Arclength, Center Angle and Half Area.

a) Radius:

The radius of the circle is determined by using the formula

Radius, $\mathrm{r}=\frac{\sqrt{\text { Area }}}{\pi}$

$\pi$ is approximately 3.142 
b) Diameter:

The diameter of the circle is determined by using the formula

Diameter, $\mathrm{d}=2 \mathrm{r}$

c) Area

The area of a circle is determined by using the formula

$$
\text { Area }=\pi r^{2}
$$

d) Arclength

The length of an arc is determined by using the formula

$$
\text { Arclength }=\mathrm{R}\left(\frac{2 \pi C}{360}\right)
$$

$C$ is the center angle of the arc, $R$ is the radius of the arc

e) Center Angle

A center angle is determined by using the formula

$$
\text { CenterAngle }=\frac{\text { Perimeter }}{360}(2 \pi \mathrm{r})
$$

f)HalfArea

$$
\begin{aligned}
& \text { Half area is determined by using the formula } \\
& \text { Half area }=\frac{\text { Areaofcircle }}{2}
\end{aligned}
$$

\section{SUPPORT VECTOR MACHINE}

Support vector machine training process is applied to analyze training data to find an optimal way to classify images into their respective classes namely PDR, NPDR or Normal.

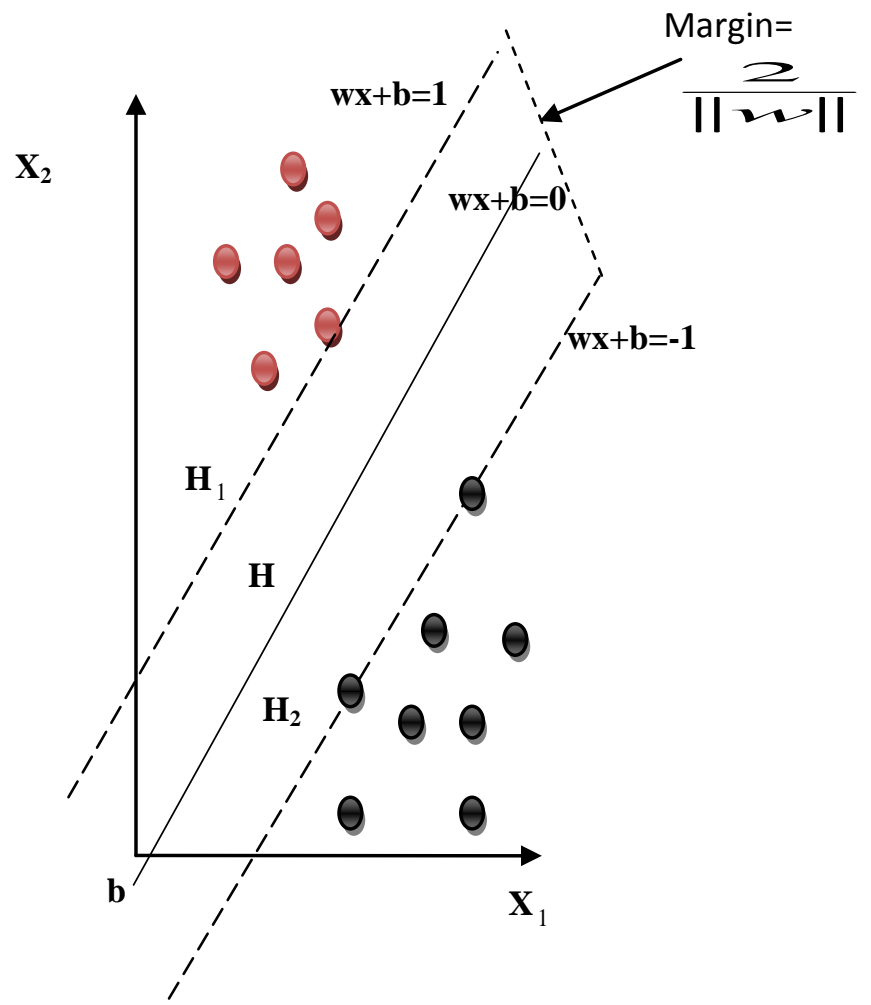

(a)

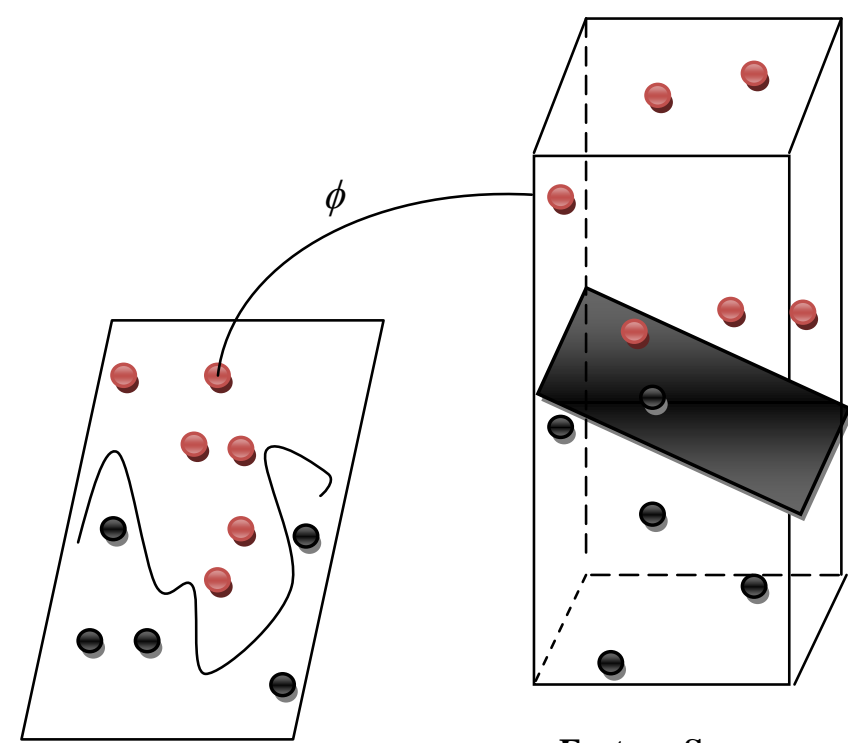

Feature Space
Input Space

(b)

Fig. 3: Architecture of SVM

SVM is a robust technique for data classification and regression. It is described in detail by Vapnik [23]. SVM models search for a hyperplane that can linearly separate classes of objects (Fig. 3 a). Support vector machine is used to discriminate the various categories. Classification parameters are calculated using support vector machine learning. The training process analyzes training data to find an optimal way to classify images into their respective classes. The training data should be sufficient to be statistically significant. The support vector machine learning algorithm is applied to produce the classification parameters according to calculated features. The derived classification parameters are used to classify the images. The image content can be discriminated into the various categories in terms of the designed support vector classifier. To fit nonlinear curves to the data, SVM make use of a kernel function to map the data into a different space where a hyperplane can be used to do the separation. SVM can be applied to non-linear classification using nonlinear kernel functions to map the input data onto a higher dimensional feature space in which the input data can be separated with a linear classifier (Fig. 3 b). Kernel function $K(\mathrm{x}, \mathrm{y})$ represents the inner product $\langle\phi(x), \phi(y)\rangle$ in feature space. In this work, we have used polynomial kernel which is given by,

$$
K\left(x, x^{\prime}\right)=\left(x \cdot x^{\prime}+1\right)^{d}
$$


Where $\mathrm{x}$ and $x^{\prime}$ are the training vectors, $\mathrm{d}$ is the kernel parameter. The size of the input training vector is $250 * 6$. The output can be one of the three categories namely normal, NPDR and PDR.

\section{PROBABILISTIC NEURAL NETWORK}

The PNN was first proposed in [24].The architecture of a typical PNN is as shown in Fig. 4. The PNN architecture is composed of many interconnected processing units or neurons organized in successive layers. The input layer unit does not perform any computation and simply distributes the input to the neurons in the pattern layer. On receiving a pattern $x$ from the input layer, the neuron $x i j$ of the pattern layer computes its output

$$
\phi_{i j}(x)=\frac{1}{(2 \pi)^{\frac{d}{2}} \sigma^{d}} \exp \left[-\frac{\left(x-x_{i j}\right)^{T}\left(x-x_{i j}\right)}{2 \sigma^{2}}\right](16)
$$

where $d$ denotes the dimension of the pattern vector $x, \sigma$ is the smoothing parameter, and $x i j$ is the neuron vector .Suppose, $\mathrm{W}_{\mathrm{dn}}$ is the input to the pattern layer for ' $\mathrm{d}$ ' varies from $1,2, \ldots$ 250 , corresponding to 250 tested images and ' $n$ ' varies from $1,2, \ldots \ldots ., 6$ corresponding to the feature vector. The pattern layer can be processed and the output layer has a node for each pattern classification. The sum for each hidden node is sent to the output layer and the highest values wins. This method has been done for three classes namely normal, NPDR and PDR.

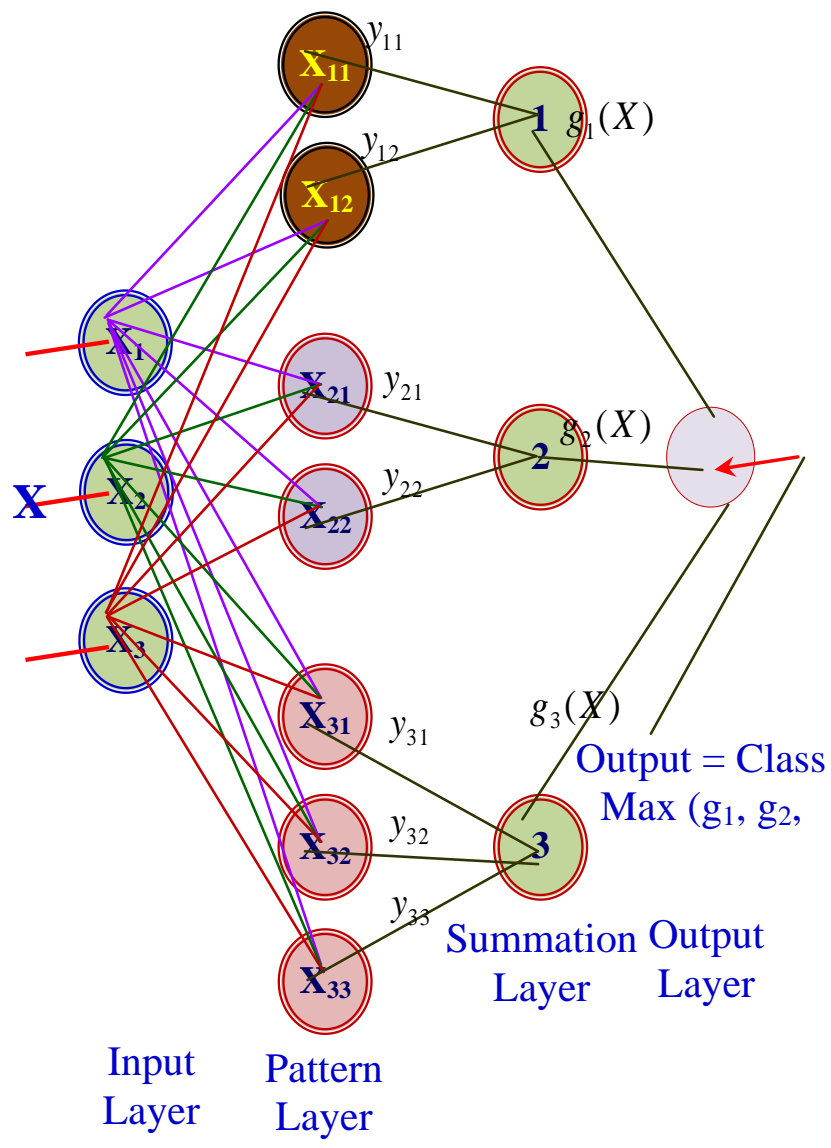

\section{EXPERIMENTAL RESULTS}

The Proposed method was implemented in Matlab and Microsoft Visual Basic 6.0 . The results of the classification Procedures are shown in Table 1. Table 2 shows the result of Sensitivity, Specificity and Percentage of accuracy for the three classes of eye images using the two classifiers. The ROC graphs are a useful tecnique for organizing classifiers and visualizing their performance. Fig. 5. gives the comparison of ROC curve for the two models. Thus SVM overwhelms the PNN model. Fig 6 - Fig 7 are the snapshots for diagnosing the retinal images as normal, Nonproliferative diabetic retinopathy or Pro-liferative retinopathy using PNN and SVM Classification

Table 1: Results of PNN and SVM classification

\begin{tabular}{|l|l|l|l|l|}
\hline Models & $\begin{array}{l}\text { True } \\
\text { Positive }\end{array}$ & $\begin{array}{l}\text { True } \\
\text { Negative }\end{array}$ & $\begin{array}{l}\text { False } \\
\text { Positive }\end{array}$ & $\begin{array}{l}\text { False } \\
\text { Negative }\end{array}$ \\
\hline PNN & 180 & 44 & 6 & 20 \\
\hline SVM & 196 & 48 & 2 & 4 \\
\hline
\end{tabular}

Table2:Results of sensitivity, specificity, \% of accuracy

\begin{tabular}{|l|l|l|l|}
\hline Models & Sensitivity & Specificity & Accuracy \\
& & & \\
\hline PNN & 90 & 88 & 89.6 \\
\hline SVM & 98 & 96 & 97.6 \\
\hline
\end{tabular}

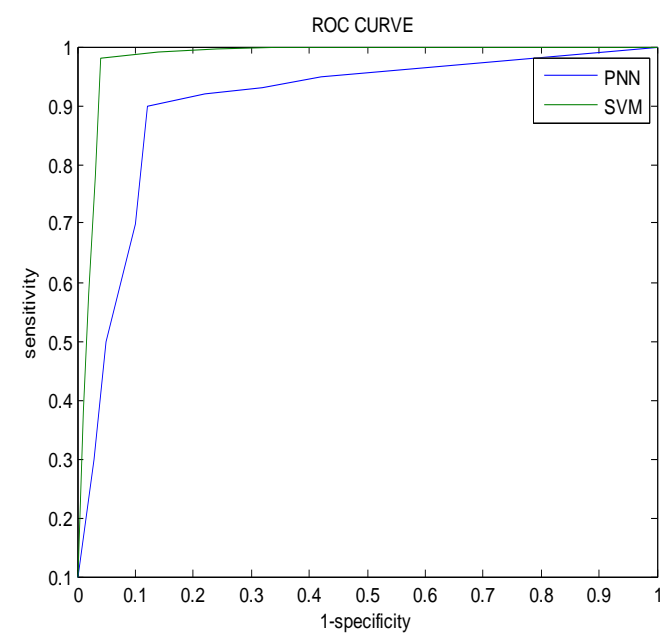

Fig. 5: ROC Curve for Comparison of PNN and SVM

Fig.4 Architecture of Probabilistic neural Network 


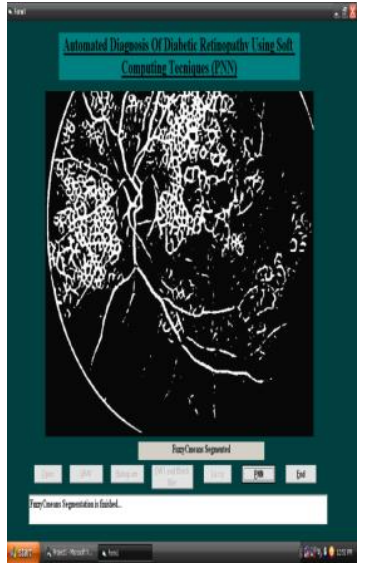

(a)PNN Training

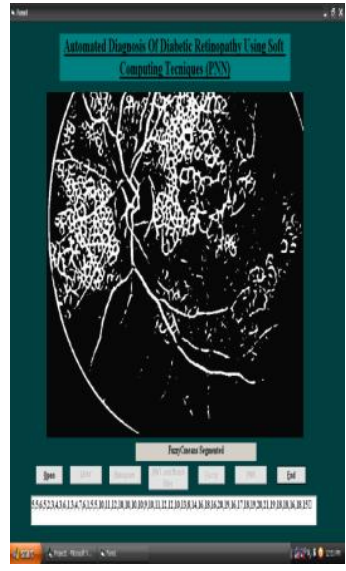

(b) PNN Testing
Fig. 6: Snapshots for PNN Classification

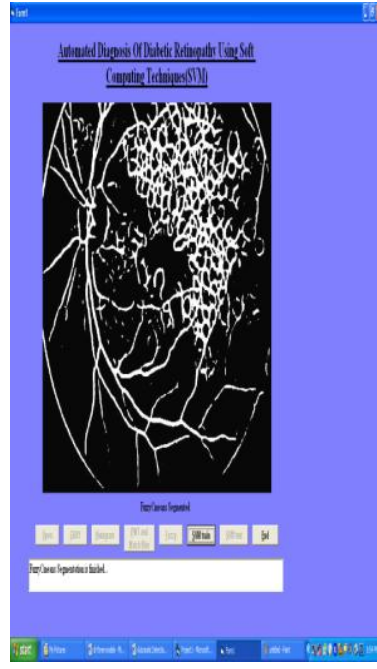

(a) SVM Training

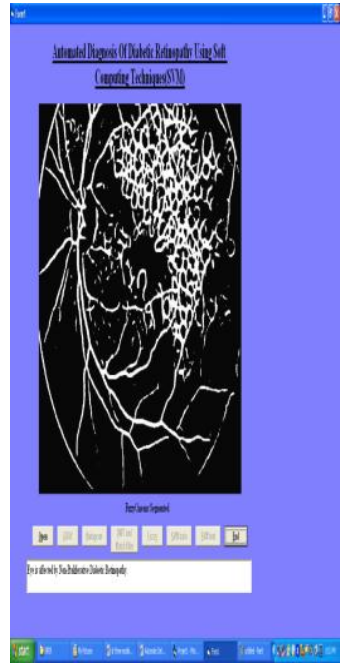

(b) SVM Testing
Fig. 7: Snapshots for SVM Classification

Clearly, the two classification system produces encouraging results. Sinthanayothin et al. [25] have differentiated diabetic retinopathy from normal retina using image processing algorithms. In their method, retinal images were preprocessed using adaptive, local, contrast enhancement. Their system, based on a multilayer perceptron neural network, yielded a sensitivity of $80.21 \%$ and a specificity of $70.66 \%$.

Larsen et al, have used image processing algorithm for the detection of hemorrhages and microaneurysms to diagnose diabetes retinopathy [26]. Their algorithm demonstrated a specificity of $71.4 \%$ and a resulting sensitivity of $96.7 \%$ in detecting diabetic retinopathy when applied at a tentative threshold setting for use in diabetic retinopathy screening.

Our system is far better as compared to the other works discussed so far. In our present work, we are able to identify normal, npdr and pdr cases of the DR correctly with an accuracy of more than $80 \%$ and a sensitivity of more than $90 \%$ in all the two models.

\section{CONCLUSION}

Diabetic Retinopathy is a disease which causes vision loss rapidly. The input color retinal images are of poor quality. So they were pre-processed using Grayscale conversion, Adaptive Histogram Equalization, Discrete Wavelet Transform, Matched filter and Fuzzy C-means segmentation. From the pre-processed images features were extracted for classification process. As an achievement of this work, the DR has been classified into two categories NPDR and PDR using PNN and SVM. All the two techniques used for the classification were good in performance, but SVM is more efficient than PNN from the obtained results. Thus this work has given a successful Diabetic Retinopathy Diagnosing method which helps to diagnose the disease in early stage which mutually reduces the manual work.

\section{ACKNOWLEDGMENT}

The authors would like to thank Dr. W. Ashok baskaran, Senior Civil Surgeon, Opthalmalogy, Cuddalore for his help in obtaining the images used in this research.

\section{REFERENCES}

[1] Diabetes http://en.wikipedia.org/wiki/Diabetes_mellitus

[2] Advanced Vision Care: http://www.advancedvisioncare.com/conditions.php

[3] Vallabha,D., Dorairaj, R., Namuduri K. and Thompson., H, "Automated Detection and Classification of Vascular Abnormalities in Diabetic Retinopathy", IEEE, 2004.

[4] R.Sivakumar, G.Ravindran, M.Muthayya, S.Lakshminarayanan, C.U. Velmurughendran, "Diabetic Retinopathy Classification", IEEE, 2003.

[5] Thomas Walter, Jean-Claude Klein, Pascale Massin, and Ali Erginay ,'A Contribution of Image Processing to the Diagnosis of Diabetic Retinopathy - Detection of Exudates in Color Fundus Images of the Human Retina", VOL. 21, NO. 10, IEEETransactions On Medical Imaging, October, 2002 ,

[6] [HT Nguyen, M Butler, A Roychoudhry, AG Shannon, J Flack, P Mitchell, "Classification of Diabetic retinopathy using neural networks", 18th Annual International Conference of the IEEE Engineering in Medicine and Biology Society, Amsterdam 1996

[7] A.M. Mendonca, A.J.Campilho, J.M. Nunes, “ Automatic Segmentation of Microaneurysms in Retinal Angiograms of Diabetic Patients".

[8] Huiqi Li and Opas Chutatape, "Fundus Image Features Extraction", Proceedings of the 22nd Annual EMBS International Conference, July 2328, 2000.

[9] María García, Roberto Hornero, Clara I. Sanchez, María I. Lopez and Ana Díez, "Feature Extraction and Selection for the Automatic Detection of Hard 
Exudates in Retinal Images", Proceedings of the 29th Annual International Conference of the IEEE EMBS Cite Internationale, Lyon, France, August 23-26, 2007.

[10] Kenneth W. Tobin, Mohamed Abdelrahman, Edward Chaum,V.Priya Govindasamy, Thomas P. Karnowski, "A Probabilistic Framework for Content-Based Diagnosis of Retinal Disease", Proceedings of the 29th Annual InternationalConference of the IEEE EMBS Cite Internationale, Lyon, France August 23-26, 2007.

[11] S .Chaudhuri, S.Chatterjee and N.Katz, " Detection of blood vessels in retinal i mages using twodimensional matched filters",IEEE Transactions on Medical Imaging,PP 263-269, 1989.

[12] Jie Tian, Shanhua Xue, Haining Huang "Classification of Underwater Objects Based on Probabilistic Neural Network", Fifth International Conference on Natural Computation, IEEE, 2009.

[13] Katia Estabridis and Rui J. P. de Figueiredo "Automatic Detection and diagnosis of diabetic retinopathy", IEEE, 2007.

[14] V. Vijaya Kumari , N. Suriyanarayanan , C.Thanka Saranya "Feature Extraction for Early Detection of Diabetic Retinopathy", International Conference on Recent Trends in Information, Telecommunication and Computing, 2010.

[15] Alireza Osareh , Majid Mirmehdi, Barry Thomas, and Richard Markham, "Classification and Localisation of Diabetic-Related Eye Disease", Springer-Verlag Berlin Heidelberg ,pp. 502- 516, 2002

[16] Jian Wu, Guangming Zhang, Yanyan Cao, and Zhiming Cui, " Research on Cerebral Aneurysm Image Recognition Method Using Bayesian Classification ", Proceedings of the 2009 International Symposium on Information Processing (ISIP'09), Huangshan, P. R. China, August 21-23, pp. 058-062, 2009.

[17] Yosawin Kangwanariyakul, Chanin Nantasenamat, Tanawut Tantimongcolwat, Thanaokorn Naenna,"
Data Mining Of Magnetocardiograms For Prediction of Ischemic Heart Disease" EXCLI Journal, 2010.

[18] Nageswara Rao Pv, Uma Devi T, Dsvgk Kaladhar,Gr Sridhar, Allam Appa Rao,” A Probabilistic Neural Network Approach Forprotein Superfamily Classification, Journal of Theoretical and Applied Information Technology.

[19] Inan Guler and Elif Derya U beyli, "Multiclass Support Vector Machinesfor EEG- Signals Classification",IEEE Transactions On Information Technology In Biomedicine, vol. 11, no. 2, March, 2007.

[20] Lili Xu, Shuqian Luo," Support Vector Macidne Based Method For Identifying Hard Exudates In Retinal Images", IEEE, 2009.

[21] Priya.R , Aruna.P, " Automated Classification System For Early Detection Of Diabetic Retinopathy In Fundus Images", International Journal Of Applied Engineering Research, Dindigul, Volume 1, No 3,2010.

[22] Fundus Camera: http://en.wikipedia.org/wiki/Fundus_camera

[23] [23] Vapnik. V, "Statistical learning theory Wiley", New York, 1998.

[24] D. F. Specht, "Probabilistic neural networks," Neural Networks, vol. 3,no. 1, PP. 109-118, 1990.

[25] C. Sinthanayothin, V. Kongbunkiat, S. Phoojaruenchanachai, A. Singalavanija,"Automated screening system for diabetic retinopathy, in: 3rd International Symposium on Image and Signal Processing and Analysis, vol. 44, no. 2, 2003, pp. 767-771.

[26] M. Larsen, J. Godt, N. Larsen, H. Lund-Andersen, A.K. Sjolie, E. Agardh, H. Kalm, M.Grunkin, D.R. Owens, Automated detection of fundus photographic red lesions in diabetic retinopathy, Investigative Ophthalmology \& Visual Science 44 (2) (2003) 761-766. 